\title{
Low Nanogram Detection of Nucleotides Using Fast Atom Bombardment-Mass Spectrometry ${ }^{1}$
}

\author{
Qing-Mei Weng,* William M. Hammargren, ${ }^{*}$ Debra Slowikowski, ${ }^{*}$ Karl H. Schram,,2 \\ Katherine Z. Borysko, $\uparrow$ Linda L. Wotring, $\dagger$ and Leroy B. Townsend $\dagger$ \\ *Department of Pharmaceutical Sciences, College of Pharmacy, University of Arizona, Tucson, Arizona 85721; and \\ †College of Pharmacy, University of Michigan, Ann Arbor, Michigan 48109
}

Received July 18, 1988

The effect of trimethylsilyl (TMS) derivatization on detection limits of mononucleotides in fast atom bombardment-mass spectrometry (FAB-MS) was examined. FAB-MS methods were developed to optimize sensitivity using adenosine $5^{\prime}$-monophosphate as a model compound and then applied to reference standards of two clinically important nucleotides: tricyclic nucleoside-5'-monophosphate (TCNMP) and 5-fluoro-2'-deoxyuridine-5'-monophosphate (FdUMP). The detection limit for the TMS derivative of TCNMP was $2.5-5 \mathrm{ng}$ / $\mu l$ and less than $2.5 \mathrm{ng} / \mu \mathrm{l}$ for FdUMP as its TMS derivative. This is greater than two orders of magnitude more sensitive than the FAB-MS analysis of the corresponding free compounds. These low detection limits for the TMS derivatives were obtained using a narrow scan range, signal averaging, detection in the negative ion mode, and 3-nitrobenzyl alcohol as the matrix. Hydrolysis of one or more of the labile TMS groups did occur, with the extent of hydrolysis being greatest in the more protic matrices. (1989 Academlc Press, Inc.

Despite the success of fast atom bombardment-mass spectrometry (FAB-MS) ${ }^{3}$ for analyzing nonvolatile compounds, such as nucleotides $(1,2)$, lack of sensitivity has been a severe restriction. Reports describing the FAB-MS analysis of nucleotides as the free compounds

\footnotetext{
${ }^{1}$ Presented in part at the 36 th American Society of Mass Spectrometry Conference, June 5-10, 1988, San Francisco.

2 To whom correspondence should be addressed.

${ }^{3}$ Abbreviations used: TMS, trimethylsilyl; FAB-MS, fast atom bombardment-mass spectrometry; TCNMP, tricyclic nucleoside5'-monophosphate; FdUMP, 5-fluoro-2'-deoxyuridine-5'-monophosphate; EI, electron impact; CI, chemical ionization; NBA, 3-nitrobenzyl alcohol; BSTFA, N,O-bis(trimethylsilyl)trifluoroacetamide; TMCS, trimethylchlorosilane; $\mathbf{M}$, molecular weight-related ion; $B$ $+\mathrm{H}$, base moiety plus hydrogen.
}

required sample levels in the mid-nanogram to micrograms per microliter range $(3,4)$. The goal of this work was to examine the effect on detection limits in FAB-MS by analysis of the trimethylsilyl derivatives of selected mononucleotides (5).

In order to optimize sensitivity, experimental conditions were determined using adenosine 5'-monophosphate (Structure 1) as a model compound. AMP was chosen because it has been studied extensively, both as the free compound and as its TMS derivative. Also, it is amenable to analysis by EI, CI, and FAB-MS $(1,5)$ and is readily available. After methods were optimized for AMP, reference standards of two clinically important nucleotides were analyzed: tricyclic nucleoside 5 '-monophosphate (TCNMP) (Structure 2) (6), a potential anticancer agent undergoing clinical trials $(7,8)$, and 5fluoro - $2^{\prime}$ - deoxyuridine - $5^{\prime}$ - monophosphate (FdUMP) (Structure 3), the active metabolite of the widely used anticancer drug, 5-fluorouracil (9).

Prior to analysis by FAB-MS, the TMS derivatives of these samples were analyzed using EI and CI to determine the number of TMS groups present.

\section{MATERIALS AND METHODS}

\section{Instrumentation}

The FAB mass spectra were obtained with a Varian MAT 311A mass spectrometer operating at an accelerating voltage of $3 \mathrm{kV}$ and resolution of 1000 . The scan rate was $11.5 \mathrm{~s} /$ decade for full scans (for samples $100 \mathrm{ng}$ or greater) and $40 \mathrm{~s} /$ decade for narrow scans (for samples less than $100 \mathrm{ng}$ ). The mass spectrometer is equipped with an Ion Tech FAB $11 \mathrm{~N}$ saddle-field atom gun (Ion Tech Ltd., Teddington, England) operated with argon at $8 \mathrm{kV}$ and $1 \mathrm{~mA}$. 3-Nitrobenzyl alcohol (NBA) (10), glycerol, and tetraglyme were used as matrices, as well as addition of crown ether to the aforementioned sol- 
<smiles>Nc1ncnc2c1ncn2COC(O)C(O)COP(=O)(O)O</smiles>

STRUCTURE 1<smiles>CN1N=C(N)c2cn(C34OC(COP(=O)(O)O)C(O)C(O3)C4O)c3ncnc1c23</smiles>

STRUCTURE 3 vent. One $\mu$ l of sample was added to one $\mu$ l matrix on the sample probe target for FAB-MS analysis.

The EI and CI mass spectra were obtained with a Finnigan MAT90 mass spectrometer operated at an accelerating voltage of $5 \mathrm{kV}$, scan rate of $1 \mathrm{~s} /$ decade, and resolution of 1000. EI conditions include an electron energy of $70 \mathrm{eV}$, an emission current of $1 \mathrm{~mA}$, and a source temperature of $200^{\circ} \mathrm{C}$. The $\mathrm{CI}$ experiments were carried out with $\mathrm{NH}_{3}$ as the reagent gas, ionized by $120 \mathrm{eV}$ electrons at an emission current of $0.2 \mathrm{~mA}$. The ionization chamber and ion source housing pressures of 1 Torr (ratio of $m / z 18 / 35=20: 1$ ) were measured at a source temperature of $150^{\circ} \mathrm{C}$.

\section{Preparation of Trimethylsilyl Derivatives}

1. Microgram samples. BSTFA (containing $1 \%$ TMCS) and pyridine (4:1) were added to the sample (50 $\mu \mathrm{g})$ and heated for $1-2 \mathrm{~h}$ at $100^{\circ} \mathrm{C}(11,12)$. The derivatized samples of AMP and TCNMP remained stable for several days following opening and resealing of the sample vial, with slow conversion to the next lower derivative. Derivatized FdUMP samples had to be analyzed within 1 day.

2. Low nanogram samples. Two microliters of sample [concentration $=5 \mathrm{ng} / \mu$ l in water] was taken to dryness under vacuum in a capillary tube, followed by addition of $1.6 \mu \mathrm{l}$ of BSTFA (containing 1\% TMCS) and $0.4 \mu \mathrm{l}$ of pyridine. After sealing, the tube was heated for $2 \mathrm{~h}$ at

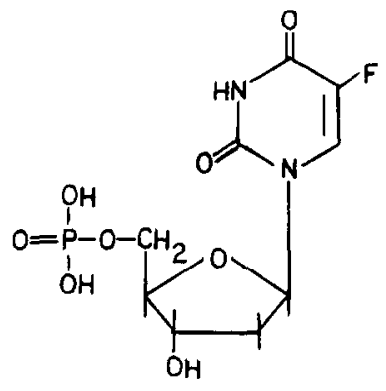

STRUCTURE 2 $100^{\circ} \mathrm{C}$. This tube was allowed to cool to room temperature and then opened and the sample analyzed.

\section{Chemicals}

The AMP and FdUMP were purchased from Sigma Chemical Co. (St. Louis, MO). TCNMP was provided by L. Townsend, L. Wotring, and K. Borysko at the University of Michigan.

3-Nitrobenzyl alcohol (NBA) and tetraglyme were obtained from Aldrich Chemical Co. (Milwaukee, WI).

BSTFA, containing $1 \%$ trimethylchlorosilane, and pyridine were purchased from Pierce Chemical Co. (Rockford, IL).

\section{RESULTS AND DISCUSSION}

Table 1 shows the results of the sensitivity studies for the selected mononucleotides analyzed by FAB-MS as both the free compound and TMS derivative. Limits of detection shown for the free compounds are those using the matrix in which the detection limits were lowest. Low detection limits for AMP(TMS $)_{3}(5 \mathrm{ng} / \mu \mathrm{l})$, TCNM$\mathrm{P}(\mathrm{TMS})_{3}(2.5-5 \mathrm{ng} / \mu \mathrm{l})$, and FdUMP(TMS $)_{2}(<2.5 \mathrm{ng} /$

TABLE 1

Sensitivity Studies of Nucleotides Using FAB-MS

\begin{tabular}{|c|c|c|c|}
\hline \multirow[b]{2}{*}{ Nucleotide } & \multirow[b]{2}{*}{ Matrix $^{a}$} & \multicolumn{2}{|c|}{ Detection limit } \\
\hline & & $\operatorname{Pos}(\mathrm{ng} / \mu \mathrm{l})$ & $\mathrm{Neg}(\mathrm{ng} / \mu \mathrm{l})$ \\
\hline \multicolumn{4}{|c|}{ I. Free } \\
\hline AMP & Glycerol & 250 & 50 \\
\hline TCNMP & Gly + cr. ether & $>500$ & 500 \\
\hline FdUMP & Glycerol & 500 & 400 \\
\hline \multicolumn{4}{|c|}{ II. TMS (NBA matrix) } \\
\hline AMP & & $5\left(\mathrm{TMS}_{4}\right)$ & $5\left(\mathrm{TMS}_{3}\right)$ \\
\hline TCNMP & & $5\left(\mathrm{TMS}_{3}\right)$ & $\left.2.5-5 \mathrm{TMS}_{3}\right)$ \\
\hline FdUMP & & $800\left(\mathrm{TMS}_{2}\right)$ & $\ll 2.5\left(\mathrm{TMS}_{2}\right)$ \\
\hline
\end{tabular}

${ }^{a}$ Only results for the matrix providing lowest detection limits are given. 


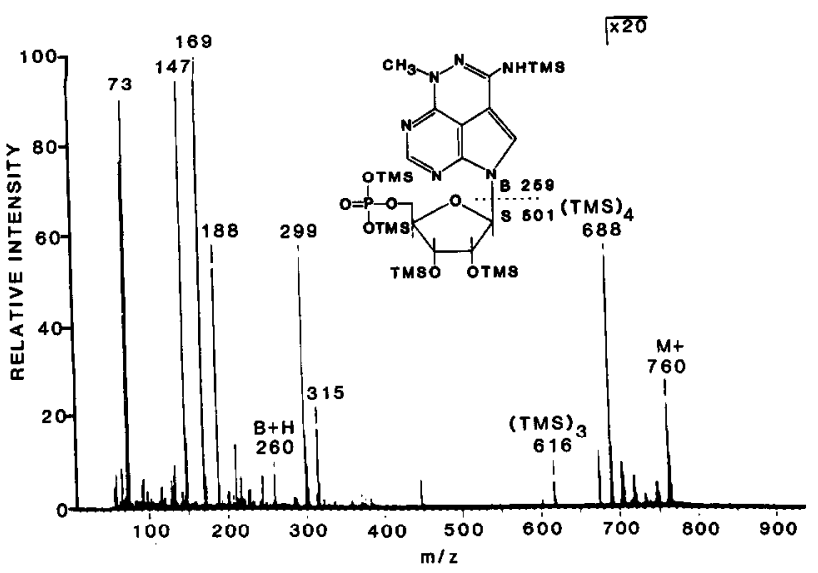

FIG. 1. EI mass spectrum of $1 \mu \mathrm{g}$ TCNMP as TMS derivative. Note that the spectrum above $m / z 700$ is multiplied by a factor of 20 .

$\mu 1)$ using FAB were obtained by analysis of the TMS derivatives with 3-nitrobenzyl alcohol as the matrix and scan averaging over a narrow mass range in the negative ion detection mode. TMS derivatization improved sensitivity over the free compound by over two orders of magnitude for TCNMP and FdUMP. Negative ion detection is more sensitive than the positive ion mode, especially for FdUMP, in which case the fluorine group presumably greatly enhances the negative ion signal.

Prior to analysis by FAB, the extent of derivatization of each of the samples was checked using EI and/or CI. For AMP, the relative abundance of the ion representing the $\mathrm{TMS}_{5}$ derivative was $5 \%$ and the $\mathrm{TMS}_{4}$ derivative was $4 \%$, indicating an approximately equal distribution of these two derivatives. Formation of the fully derivatized $\mathrm{TMS}_{5}(m / z 760)$ product for TCNMP occurred to only a minor extent, approximately $1 \%$, with the $\mathrm{TMS}_{4}$ $(\mathrm{m} / z 688)$ analog being the major species, $91 \%$, followed by the $\mathrm{TMS}_{3}(m / z 616)$ at $8 \%$ (Fig. 1). Other ions of interest (12) in the EI spectrum of TCNMP include phosphate related ions at $m / z 299$ and 315 , a sugar ion at $\mathrm{m} / z 169$ which is the base peak of the spectrum, and ions of the aglycone with $(m / z 260)$ and without $(m / z 188)$ a TMS group on the exocyclic amino group. In the case of FdUMP, using CI, the $\mathrm{TMS}_{3}(\mathrm{~m} / z$ 470) derivative represents $98 \%$ of the identified products and the $\mathrm{TMS}_{4}(\mathrm{~m} / z$ 542 ) only $2 \%$ (Fig. 2). Thus, in each of the derivatization reactions of these nucleotides, a mixture of products is formed in which the product having one less than a full complement of TMS groups predominates.

Detection limits, with a signal to noise ratio of at least 2, for the derivatized analogs of TCNMP and FdUMP were, respectively, $100 \mathrm{ng}$ and $1 \mu \mathrm{g}$ in EI and $1 \mu \mathrm{g}$ for either compound in $\mathrm{CI}$.

Figure 3A shows the negative ion FAB-MS spectrum of $500 \mathrm{ng} / \mu \mathrm{l}$ TCNMP as its TMS derivative using NBA matrix. Significant ions in this spectrum include the base peak at $m / z 615$, corresponding to the $(\mathrm{M}-\mathrm{H})^{-}$ion

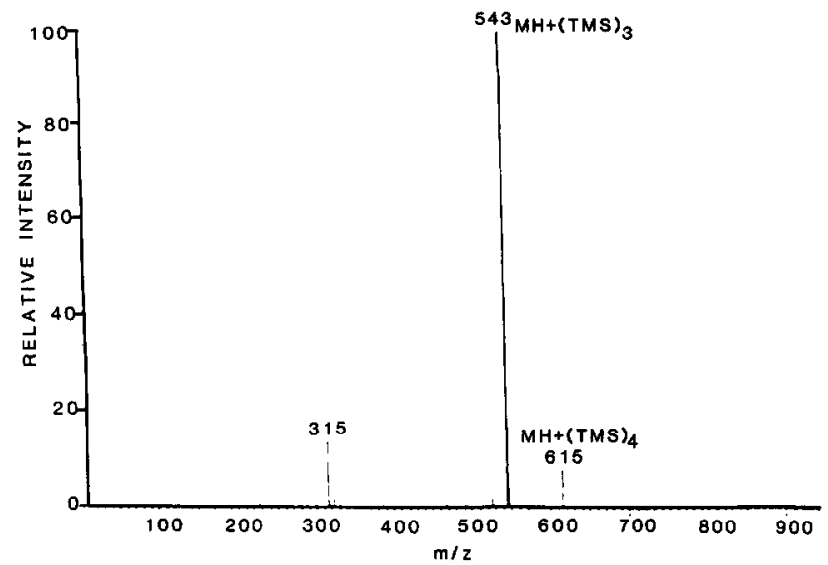

FIG. 2. CI mass spectrum of $1 \mu \mathrm{g}$ FdUMP as TMS derivative using ammonia reagent gas.

of TCNMP containing three TMS groups, with its NBA-related adduct ion occurring at $\mathrm{m} / z$ 752. The $\mathrm{TMS}_{2}$ derivative of TCNMP is represented by the peak at $m / z 543$. The peak at $m / z 169$ corresponds to the sugar moiety without the phosphate and contains one TMS
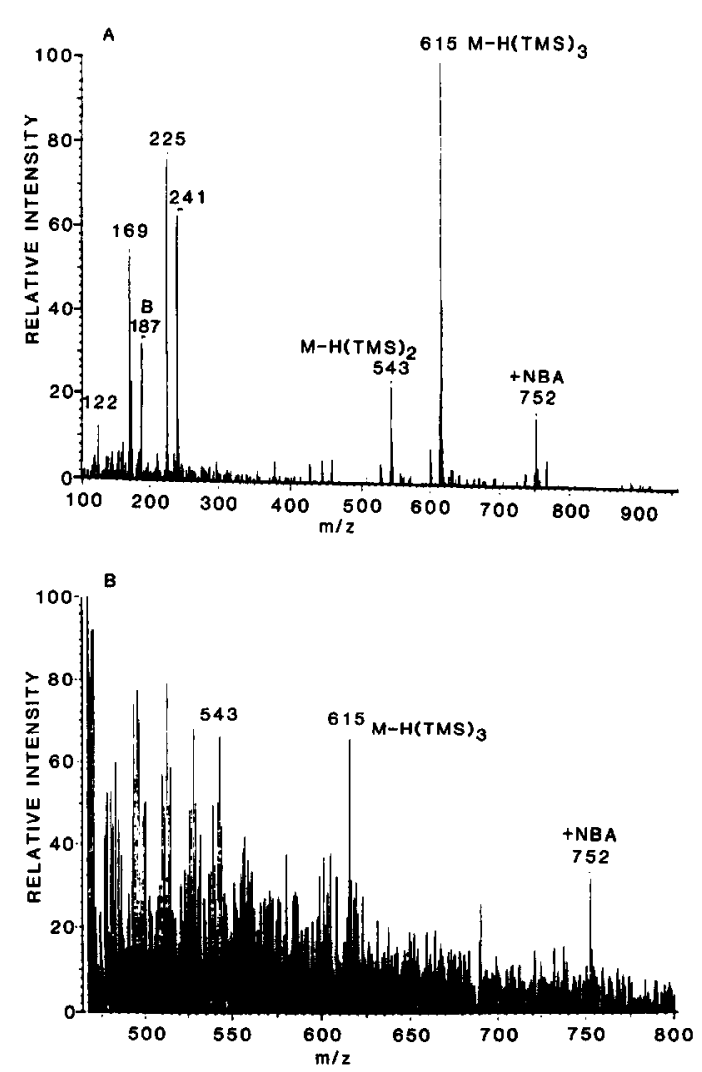

FIG. 3. Negative ion FAB-MS spectra of (A) 500 and (B) $2.5 \mathrm{ng} / \mu \mathrm{l}$ of TCNMP as TMS derivative using NBA matrix. Base peak represents the $\mathrm{TMS}_{3}$ derivative. The peak at $m / z 752$ is an NBA-related adduct of the $\mathrm{TMS}_{3}$ derivative of TCNMP (see Ref. (13) for a discussion of the mass spectrometry of NBA). 

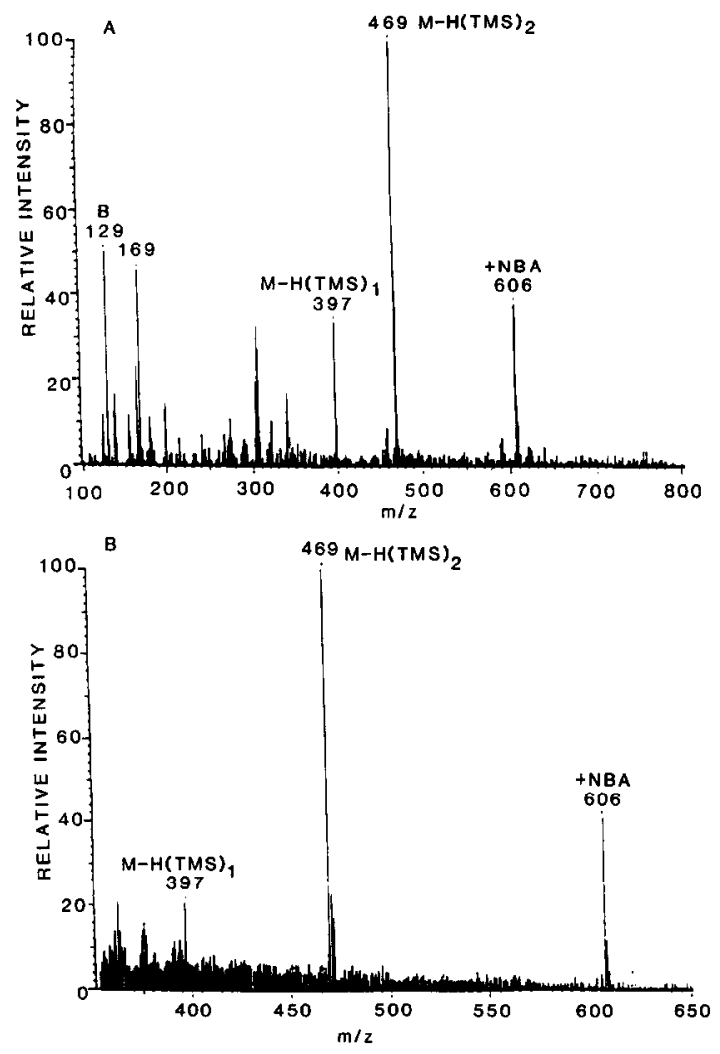

FIG. 4. Negative ion FAB-MS spectra of (A) 500 and (B) $2.5 \mathrm{ng} / \mu \mathrm{l}$ of FdUMP as TMS derivative using NBA matrix. Base peak represents the $\mathrm{TMS}_{2}$ derivative. The peak at $m / z 606$ is an NBA-related adduct of the TMS ${ }_{2}$ derivative of FdUMP (see Ref. (13) for a discussion of the mass spectrometry of NBA).

group. The peak at $m / z 187$ represents the base without TMS groups. The peak at $m / z 225$, and tentatively 241 , corresponds to the derivatized phosphate group (12). Figure 3B shows the negative ion FAB-MS spectrum of the molecular ion region of $2.5 \mathrm{ng} / \mu \mathrm{l}$ TCNMP as its $\mathrm{TMS}_{3}$ derivative using NBA matrix.

Figure 4A shows the negative ion FAB-MS spectrum of $500 \mathrm{ng} / \mu \mathrm{l}$ FdUMP as its TMS derivative using NBA matrix. The base peak at $m / z 469$ represents the (M$\mathrm{H})^{-}$ion of FdUMP containing two TMS groups, with its NBA-related adduct ion occurring at $m / z 606$. The peak at $m / z 129$ corresponds to the base moiety without TMS groups, with the peak at $m / z 169$ sugar-related. In Fig. $4 \mathrm{~B}$, the negative ion FAB-MS spectrum of the molecular ion region of $2.5 \mathrm{ng} / \mu \mathrm{l}$ FdUMP as its $\mathrm{TMS}_{2}$ derivative using the NBA matrix is shown. Obviously, significantly lower detection limits should be possible with this compound.

Tables 2 and 3 show the summary of results of TMS derivatization of TCNMP and FdUMP, respectively, using mass spectrometry with various ionization conditions.

Obvious from the FAB spectra of the derivatized nucleotides is that hydrolysis of one or more of the labile
TABLE 2

Summary of TCNMP TMS Derivatization: Different Ionization Conditions and Matrices $^{a}$

\begin{tabular}{cccccc}
\hline & & \multicolumn{4}{c}{ Relative intensity (\%) } \\
\cline { 3 - 6 } Ionization mode & Matrix & TMS $_{2}$ & TMS $_{3}$ & TMS $_{4}$ & TMS $_{5}$ \\
\hline EI & - & - & 8 & 91 & 8 \\
CI $\left(\mathrm{NH}_{3}\right)$ & - & - & - & 100 & 22 \\
FAB $(+)$ & Glycerol & 0.8 & 0.7 & - & - \\
FAB $(-)$ & Glycerol & 5 & 16 & - & - \\
FAB $(+)$ & NBA & 30 & 84 & - & - \\
FAB $(-)$ & NBA & 25 & 100 & - & - \\
FAB $(+)$ & Tetraglyme & - & 1.8 & 7 & 2 \\
FAB $(-)$ & Tetraglyme & - & 18 & 78 & 16 \\
\hline
\end{tabular}

${ }^{a}$ All analyses were done with $1 \mu \mathrm{g}$ of sample as TMS derivative as described under Materials and Methods. Data were obtained over a full scan range $(m / z 100-1000)$.

TMS groups is occurring, with the extent of hydrolysis being greatest when glycerol is used as the matrix. Hydrolysis of the TMS group(s) must be a result of either the protic matrix or the $\mathrm{FAB}$ process itself since the $\mathrm{EI} /$ CI data show the dominant species to be the TCNMP$(\mathrm{TMS})_{4}$ and FdUMP(TMS) ${ }_{3}$ derivatives. Using NBA as the matrix, hydrolysis of one TMS group was observed, while the nonprotic tetraglyme usually displayed no hydrolytic effects; i.e., the spectra of AMP and TCNMP paralleled the EI spectra. However, routine use of tetraglyme is difficult because of its volatility. Therefore, NBA was chosen as the routine matrix for analysis of the TMS-derivatized nucleotides because it gave good sensitivity and results are reproducible. Hydrolysis appears to involve only one of the TMS groups and, once the lower derivative is formed, additional products are not observed.

TABLE 3

Summary of FdUMP TMS Derivatization: Different Ionization Conditions and Matrices ${ }^{a}$

\begin{tabular}{cccccc}
\hline & & \multicolumn{4}{c}{ Relative intensity (\%) } \\
\cline { 3 - 6 } Ionization mode & Matrix & TMS $_{1}$ & TMS $_{2}$ & TMS $_{3}$ & TMS $_{4}$ \\
\hline EI & - & - & - & $2(\mathrm{M}-15)$ & - \\
CI $\left(\mathrm{NH}_{3}\right)$ & - & - & - & 98 & 2 \\
FAB(+) & Glycerol & - & 1 & - & - \\
FAB(-) & Glycerol & 12 & 12 & - & - \\
FAB(+) & NBA & - & - & 60 & - \\
FAB(-) & NBA & - & 100 & - & - \\
\hline
\end{tabular}

${ }^{a}$ All analyses were done on $1 \mu \mathrm{g}$ of sample as TMS derivative as described under Materials and Methods. Data were obtained over a full scan range $(m / z \quad 100-800)$. Tetraglyme was not used with this sample. 
Because of the significant increase in sensitivity gained by analysis of the TMS derivatives of nucleotides by FAB mass spectrometry, the potential of quantitation of low levels of nucleotides is obvious. Experiments are currently underway to improve the technique by use of nonprotic matrices which will, hopefully, eliminate the hydrolysis problem. Other derivatives are also being examined in an effort to further lower the detection limits and to provide derivatives which are more stable to hydrolysis.

In conclusion, the FAB-MS analysis of nucleotide TMS derivatives affords a significant lowering of the detection limits compared to analysis of the free compounds. The capability of detecting or, possibly, quantitating low nanogram levels of therapeutically important nucleotide analogs, such as TCNMP and FdUMP, may permit the application of FAB-MS to biomedically important problems involving nucleic acid chemistry.

\section{ACKNOWLEDGMENT}

This work was supported by NIH Grant CA-42309.

\section{REFERENCES}

1. Slowikowski, D. L., and Schram, K. H. (1985) Nucleosides Nucleotides 4, 309-345.
2. Schram, K. H. (1988) Trends Anal. Chem. 7, 28-32.

3. Moser, H., and Wood, G. W. (1988) Biomed. Environ. Mass Spectrom. 15, 547-551.

4. Schulten, H. R., and Schiebel, H. M. (1985) Fresenius Z. Anal. Chem. 321, 531-534.

5. Schram, K. H., and Slowikowski, D. L. (1986) Biomed. Environ. Mass Spectrom. 13, 263-264.

6. Schram, K. H., and Townsend, L. B. (1971) Tetrahedron Lett. 49, 4757-4760.

7. O'Connell, M. J., Rubin, J., Hahn, R. G., Kvols, L. K., and Moertel, C. (1987) Cancer Treat. Rep. 71, 333-334.

8. Behrens, B. C., Hamilton, T. C., Louie, K. G., Grotzinger, K. R., McKoy, W. M., Tsuruo, T., Yound, R. C., and Ozols, R. F. (1986) Invest. New Drugs 4, 295-304.

9. Heidelberger, C. (1974) in Handbook of Experimental Pharmacology (Sortelli, A. C., and Johns, D. C., Eds.), pp. 193-231, SpringerVerlag, New York.

10. Meili, J., and Seibl, J. (1984) Org. Mass Spectrom. 19, 581-583.

11. Schram, K. H., and McCloskey, J. A. (1979) in GLC and HPLC Determination of Therapeutic Agents (Tsuji, K., Ed.), Part 3 pp. 1149-1190, Dekker, New York.

12. Lawson, A. M., Stillwell, R. N., Tacker, M. M., Tsuboyama, K., and McCloskey, J. A. (1971) J. Amer. Chem. Soc. 93, 1014-1024.

13. Reddy, A. M., Mykytyn, V. V., and Schram, K. H. (1988) Biomed. Environ. Mass Spectrom., in press. 